PROCEEDINGS OF THE

AMERICAN MATHEMATICAL SOCIETY

Volume 138, Number 5, May 2010, Pages 1537-1548

S 0002-9939(10)10283-4

Article electronically published on January 19, 2010

\title{
ALGEBRAIC APPROXIMATION OF GERMS OF REAL ANALYTIC SETS
}

\author{
M. FERRAROTTI, E. FORTUNA, AND L. WILSON
}

(Communicated by Daniel Ruberman)

\begin{abstract}
Two subanalytic subsets of $\mathbb{R}^{n}$ are $s$-equivalent at a common point, say $O$, if the Hausdorff distance between their intersections with the sphere centered at $O$ of radius $r$ goes to zero faster than $r^{s}$. In the present paper we investigate the existence of an algebraic representative in every $s$ equivalence class of subanalytic sets. First we prove that such a result holds for the zero-set $V(f)$ of an analytic map $f$ when the regular points of $f$ are dense in $V(f)$. Moreover we present some results concerning the algebraic approximation of the image of a real analytic map $f$ under the hypothesis that $f^{-1}(O)=\{O\}$.
\end{abstract}

\section{INTRODUCTION}

Two subanalytic subsets of $\mathbb{R}^{n}$ are $s$-equivalent at a common point, say $O$, if the Hausdorff distance between their intersections with the sphere centered at $O$ of radius $r$ goes to zero faster than $r^{s}$. This equivalence relation was introduced in [FFW], where we proved that for any real $s \geq 1$, every $s$-equivalence class of semialgebraic sets has an algebraic representative; i.e. every semialgebraic set can be approximated of order $s$ (in the previous sense) by an algebraic one.

In the present paper we investigate the existence of an algebraic representative in every $s$-equivalence class of subanalytic sets. For $s=1$ the answer is positive for all semianalytic sets, a result which cannot be extended to the class of subanalytic sets. In general, since an analytic set $X$ is locally the zero-set of an analytic map $f$, it is natural to try to approximate $X$ algebraically by means of the zero-set of a suitable truncation of the Taylor series of $f$. We prove that such a result holds when the regular points of $f$ are dense in its zero-set.

If we say that two analytic maps are $V_{s}$-equivalent if their zero-sets are $s$ equivalent, then the previous result can be seen as a jet-sufficiency theorem for $V_{s}$-equivalence. Recall that two maps have the same $k$-jet at $O$ if their derivatives up to order $k$ are the same at $O$; this jet is called sufficient with respect to some equivalence relation if all representatives of the jet are equivalent. Thus our result can be reformulated by saying that if the regular points of $f$ are dense in $V(f)=f^{-1}(O)$, then some jet of $f$ is $V_{s}$-sufficient.

Received by the editors January 9, 2009.

2000 Mathematics Subject Classification. Primary 14P15, 32B20, 32S05.

This research was partially supported by M.I.U.R. and by G.N.S.A.G.A

(C)2010 American Mathematical Society Reverts to public domain 28 years from publication 
In the final section we present some results concerning the algebraic approximation of the image of a real analytic map $f$ under the hypothesis that $f^{-1}(O)=\{O\}$. In particular we show that the algebraic approximation holds for all one-dimensional subanalytic sets, for analytic surfaces in $\mathbb{R}^{3}$, and for those analytic sets whose complexification has $O$ as a quasi-ordinary singular point. Observe that, once more, these results are jet-sufficiency theorems with respect to considering two analytic maps to be equivalent if their images are $s$-equivalent.

Note that analogous approximation results for complex analytic sets have been obtained by M. Bilski (see [B1] and [B2]) using techniques which are peculiar to the complex setting.

\section{Some Properties of $s$-EQUIVALENCE}

Let $A$ and $B$ be nonempty compact subsets of $\mathbb{R}^{n}$. Let us denote by $D(A, B)$ the classical Hausdorff distance, i.e.

$$
D(A, B)=\inf \left\{\epsilon \mid A \subseteq N_{\epsilon}(B), B \subseteq N_{\epsilon}(A)\right\},
$$

where $N_{\epsilon}(A)=\left\{x \in \mathbb{R}^{n} \mid d(x, A)<\epsilon\right\}$ and $d(x, A)=\inf _{y \in A}\|x-y\|$.

If we let $\delta(A, B)=\sup _{x \in B} d(x, A)$, then $D(A, B)=\max \{\delta(A, B), \delta(B, A)\}$.

We will denote by $\operatorname{Der}(A)$ the set of nonisolated points of $A$ and by $S_{r}$ the sphere of radius $r$ centered at the origin.

Definition 2.1. Let $A$ and $B$ be closed subanalytic subsets of $\mathbb{R}^{n}$ with $O \in$ $\operatorname{Der}(A) \cap \operatorname{Der}(B)$ and let $s$ be a real number $\geq 1$.

(1) We say that $A \leq_{s} B$ if $\lim _{r \rightarrow 0} \frac{\delta\left(B \cap S_{r}, A \cap S_{r}\right)}{r^{s}}=0$.

(2) We say that $A$ and $B$ are $s$-equivalent (and we will write $A \sim_{s} B$ ) if $A \leq_{s} B$ and $B \leq_{s} A$, i.e. if $\lim _{r \rightarrow 0} \frac{D\left(A \cap S_{r}, B \cap S_{r}\right)}{r^{s}}=0$.

It is easy to check that $\leq_{s}$ is transitive and that $\sim_{s}$ is an equivalence relationship.

As announced in the introduction, we are interested in investigating for each $s \geq 1$ the existence of an algebraic subset $Y$ which is $s$-equivalent to a given subanalytic set $A$; in this case we also say that $Y$ approximates $A$ to order $s$.

The analogous problem for semialgebraic sets was solved in [FFW]:

Theorem 2.2. For any real number $s \geq 1$ and for any closed semialgebraic set $A \subset \mathbb{R}^{n}$ of codimension $\geq 1$ with $O \in \operatorname{Der}(A)$ there exists an algebraic subset $Y$ of $\mathbb{R}^{n}$ such that $A \sim_{s} Y$.

As a consequence of the previous theorem we easily obtain that algebraic approximation to order 1 holds for all closed semianalytic sets of positive codimension:

Corollary 2.3. For any closed semianalytic set $A \subset \mathbb{R}^{n}$ of codimension $\geq 1$ with $O \in \operatorname{Der}(A)$ there exists an algebraic subset $Y$ of $\mathbb{R}^{n}$ such that $A \sim_{1} Y$.

Proof. Arguing as in [FFW, Lemma 1.1], if $C(A)$ denotes the tangent cone at $O$ to $A$, we have that $C(A) \sim_{1} A$. By $\mathrm{KR}$, Lemma 1.2], $C(A)$ is a semianalytic cone; hence it is semialgebraic (see for instance $[\mathrm{H}]$ ). Then by Theorem 2.2 there exists an algebraic subset $Y$ of $\mathbb{R}^{n}$ such that $C(A) \sim_{1} Y$ and hence $A \sim_{1} Y$.

On the other hand the result of Corollary 2.3 cannot be extended to the class of subanalytic sets. Let $B$ be a compact semianalytic set in $\{1\} \times \mathbb{R}^{n-1}$ which is not semialgebraic. Let $A$ be the cone over $B$ in $\mathbb{R}^{n}$ (with vertex at the origin); $A$ is subanalytic but not semialgebraic. If there exists an algebraic set $Y$ such that 
$A \sim_{1} Y$, then arguing as in [FFW, Proposition 1.3] we have that $A=C(A)=C(Y)$ in contradiction with the fact that $C(Y)$ is semialgebraic.

Before presenting the results obtained for analytic sets, we will devote the final part of this section to introduce some technical tools useful to test $s$-equivalence, which are partly used already in FFW and partly new.

For any subanalytic subset $A \subset \mathbb{R}^{n}, O \in \operatorname{Der}(A)$, and for any real positive constant $\sigma$, we will denote

$$
\mathcal{U}(A, \sigma)=\left\{x \in \mathbb{R}^{n} \mid \exists y \in A \cap S_{\|x\|},\|x-y\|<\|x\|^{\sigma}\right\} .
$$

Arguing as in [FFW, Proposition 2.4] we obtain:

Proposition 2.4. Let $A, B$ be closed subanalytic subsets of $\mathbb{R}^{n}$ and let $s \geq 1$. If $O \in \operatorname{Der}(A) \cap \operatorname{Der}(B)$, then $A \leq_{s} B$ if and only if there exist real constants $R>0$ and $\sigma>s$ such that $A \cap B(O, R) \backslash\{O\} \subseteq \mathcal{U}(B, \sigma)$.

We can more simply test the $s$-equivalence of two subanalytic sets by means of the following "horn-neighborhoods":

$$
\mathcal{H}(A, \sigma)=\left\{x \mid d(x, A)<\|x\|^{\sigma}\right\} .
$$

We will prove this fact using the following geometric result:

Lemma 2.5. Let $\left\{P_{i}\right\},\left\{Q_{i}\right\}$ and $\left\{T_{i}\right\}$ be sequences of points in $\mathbb{R}^{n}$ converging to $O$ when $i \rightarrow \infty$ and let $\tau>1$. Assume that

(1) $\left\|P_{i}\right\|=\left\|Q_{i}\right\|=r_{i}$;

(2) $\lim _{i \rightarrow \infty} \frac{\left\|P_{i}-T_{i}\right\|}{r_{i}^{\tau}}=0$;

(3) $\lim _{i \rightarrow \infty} \angle\left(T_{i}, O, Q_{i}\right)=0$, where $\angle\left(T_{i}, O, Q_{i}\right)$ denotes the convex angle determined by the half lines $O T_{i}$ and $O Q_{i}$;

(4) the sequence $\angle\left(O, T_{i}, Q_{i}\right)$ does not converge to $\pi / 2$.

Then $\lim _{i \rightarrow \infty} \frac{\left\|P_{i}-Q_{i}\right\|}{r_{i}^{\tau}}=0$.

Proof. If the thesis fails to hold, then up to choosing suitable subsequences we can assume that $\frac{\left\|P_{i}-Q_{i}\right\|}{r_{i}^{T}} \geq C$ for some constant $C>0$.

For each $i$ let $\Delta_{i}$ denote the tetrahedron obtained from the tetrahedron with vertices $O, P_{i}, Q_{i}, T_{i}$ through the homothety with scaling factor $1 / r_{i}^{\tau}$. By the contradiction hypothesis, the length of the edge of $\Delta_{i}$ with vertices $\frac{P_{i}}{r_{i}^{\tau}}$ and $\frac{Q_{i}}{r_{i}^{\tau}}$ is at least $C>0$.

By hypothesis (2) the length of the edge in $\Delta_{i}$ with vertices $\frac{P_{i}}{r_{i}^{\tau}}$ and $\frac{T_{i}}{r_{i}^{\tau}}$ goes to 0 , hence the angle $\angle\left(P_{i}, Q_{i}, T_{i}\right) \rightarrow 0$.

Moreover $\angle\left(P_{i}, O, T_{i}\right) \rightarrow 0$ : namely in the triangle with vertices $O, \frac{P_{i}}{r_{i}}, \frac{T_{i}}{r_{i}}$ the edge with vertices $O$ and $\frac{P_{i}}{r_{i}}$ has length 1 , while the length of the edge with vertices $\frac{P_{i}}{r_{i}}$ and $\frac{T_{i}}{r_{i}}$ converges to 0 because $\frac{\left\|P_{i}-T_{i}\right\|}{r_{i}} \leq \frac{\left\|P_{i}-T_{i}\right\|}{r_{i}^{T}}$ for $i$ big enough.

By hypothesis (3) also the angle $\angle\left(P_{i}, O, Q_{i}\right) \rightarrow 0$. Hence, by (1), the angle $\angle\left(O, P_{i}, Q_{i}\right) \rightarrow \pi / 2$.

On the other hand, by the previous considerations, the two angles $\angle\left(O, P_{i}, Q_{i}\right)$ and $\angle\left(O, T_{i}, Q_{i}\right)$ have the same limit when $i \rightarrow \infty$, in contradiction with the hypothesis (4).

Proposition 2.6. Let $A, B$ be closed subanalytic subsets of $\mathbb{R}^{n}$ and let $s \geq 1$. If $O \in \operatorname{Der}(A) \cap \operatorname{Der}(B)$, then $A \leq_{s} B$ if and only if there exist real constants $R>0$ and $\sigma>s$ such that $A \cap B(O, R) \backslash\{O\} \subseteq \mathcal{H}(B, \sigma)$. 
Proof. Since $\mathcal{U}(B, \sigma) \subseteq \mathcal{H}(B, \sigma)$, by Proposition 2.4, if $A \leq_{s} B$, then $A \cap B(O, R) \backslash$ $\{O\} \subseteq \mathcal{H}(B, \sigma)$ for some $R>0$ and $\sigma>s$.

Conversely suppose that $A \cap B(O, R) \backslash\{O\} \subseteq \mathcal{H}(B, \sigma)$ for some $R>0$ and $\sigma>s$; we can assume $R<1$. For a contradiction assume that $A \leq_{s} B$ does not hold, i.e. that $A \cap B(O, r) \backslash\{O\} \nsubseteq \mathcal{U}(B, \tau)$ for all $\tau>s$ and for all $r>0$. Choose $\tau$ such that $s<\tau<\sigma$. Then $Y=(A \cap B(O, R)) \backslash \mathcal{U}(B, \tau)$ is subanalytic and contains points arbitrarily close to $O$. By the curve selection lemma there is an analytic curve $\alpha:[0, \epsilon) \rightarrow Y$ such that $\alpha(0)=O$. Then $d(\alpha(t), B)<\|\alpha(t)\|^{\sigma}$ and $d\left(\alpha(t), B \cap S_{\|\alpha(t)\|}\right) \geq\|\alpha(t)\|^{\tau}$ for $t>0$.

The set $Z=\left\{y \in B \mid \exists t \in[0, \epsilon)\right.$ such that $\left.d(\alpha(t), y)<\|\alpha(t)\|^{\sigma}\right\}$ is subanalytic and such that $O \in \operatorname{Der}(Z)$. Let $u$ be a unit vector in the tangent cone to $Z$ at $O$; by $[\mathrm{KR}$ there is an analytic curve $\beta$ in $Z$ such that $\beta(0)=O$ and such that the image of $\beta$ is tangent to $u$ at $O$.

Let $\left\{T_{i}\right\}$ be a sequence of points in the curve $\beta$ converging to $O$. By construction for each $i$ there exists a point $P_{i}$ in the curve $\alpha$ such that $\left\|P_{i}-T_{i}\right\|<\left\|P_{i}\right\|^{\sigma}$.

The sequence $\left\{P_{i}\right\}$ converges to $O$; otherwise, by compactness and up to choosing a subsequence, $\left\{\left\|P_{i}\right\|\right\}$ would converge to some positive real number $\lambda \leq R<1$. Since $0 \leq\left\|P_{i}\right\|-\left\|P_{i}\right\|^{\sigma}<\left\|T_{i}\right\|$, we would get $\lambda \in\{0,1\}$, which is a contradiction.

Then $\lim _{i \rightarrow \infty} \frac{\left\|P_{i}-T_{i}\right\|}{\left\|P_{i}\right\|^{\tau}}=0$.

For each $i$ big enough, there exists a point $Q_{i}$ in the curve $\beta$ with $\left\|P_{i}\right\|=\left\|Q_{i}\right\|$. Then $\left\|P_{i}-Q_{i}\right\| \geq\left\|P_{i}\right\|^{\tau}$. On the other hand, since $Q_{i}$ and $T_{i}$ lie in the analytic curve $\beta$, we have that $\lim _{i \rightarrow \infty} \angle\left(T_{i}, O, Q_{i}\right)=0$, and the sequence $\angle\left(O, T_{i}, Q_{i}\right)$ converges either to 0 or to $\pi$. Thus the sequences $\left\{P_{i}\right\},\left\{Q_{i}\right\},\left\{T_{i}\right\}$ satisfy all the hypotheses of Lemma 2.5, and hence $\lim _{i \rightarrow \infty} \frac{\left\|P_{i}-Q_{i}\right\|}{\left\|P_{i}\right\| T^{\tau}}=0$, which is a contradiction.

As an immediate consequence of Proposition 2.4 or Proposition 2.6 we have that:

Proposition 2.7. Let $A, A^{\prime}, B$ and $B^{\prime}$ be closed subanalytic subsets of $\mathbb{R}^{n}$, and assume that $O$ is not isolated in any of them.

(1) If $A \leq_{s} B$ and $A^{\prime} \leq_{s} B^{\prime}$, then $A \cup A^{\prime} \leq_{s} B \cup B^{\prime}$.

(2) If $A \sim_{s} B$ and $A^{\prime} \sim_{s} B^{\prime}$, then $A \cup A^{\prime} \sim_{s} B \cup B^{\prime}$.

We will also need the following result, which can be proved by arguing as in FFW, Corollary 2.6]:

Proposition 2.8. Let $A \subset B \subseteq \mathbb{R}^{n}$ be closed subanalytic sets with $O \in \operatorname{Der}(A)$ and $\overline{B \backslash A}=B$. For any $s \in \mathbb{R}, s \geq 1$, there exists a positive $R$ and a closed subanalytic set $\Gamma \subseteq B$ such that $\Gamma \cap A \cap B(O, R)=\{O\}$ and $B \leq_{s} \Gamma$.

\section{Some technical tools}

Let $L: \mathbb{R}^{n} \rightarrow \mathbb{R}^{p}$ be a linear map. Following [TW], let us consider the function d: $\operatorname{Hom}\left(\mathbb{R}^{n}, \mathbb{R}^{p}\right) \rightarrow \mathbb{R}$ defined by

$$
\mathbf{d}(L)= \begin{cases}0 & \text { if } \operatorname{rk} L<p, \\ \inf _{v \perp \text { ker } L,\|v\|=1}\|L(v)\| & \text { if } \operatorname{rk} L=p .\end{cases}
$$

Evidently $\mathbf{d}(L)>0$ if and only if $L$ is surjective.

As usual we endow $\operatorname{Hom}\left(\mathbb{R}^{n}, \mathbb{R}^{p}\right)$ with the standard norm

$$
\|L\|=\max _{u \neq 0} \frac{\|L(u)\|}{\|u\|} \text {. }
$$


We will prove that the function $\mathbf{d}: \operatorname{Hom}\left(\mathbb{R}^{n}, \mathbb{R}^{p}\right) \rightarrow \mathbb{R}$ is uniformly continuous w.r.t. the previous norm by using the following lemmas:

Lemma 3.1. Let $L: \mathbb{R}^{n} \rightarrow \mathbb{R}^{p}$ be a linear map, with $n \geq p$, and let $E(L)$ denote the frontier of $L\left(S^{n-1}\right)$. Then $\mathbf{d}(L)=d(O, E(L))$.

Proof. Assume that $L$ is surjective. If $n>p$, then $L\left(S^{n-1}\right)$ is a solid ellipsoid in $\mathbb{R}^{p}$, while if $n=p$, we have that $L\left(S^{n-1}\right)=E(L)$. Thus in both cases $E(L)=$ $L\left(S^{n-1} \cap(\operatorname{ker} L)^{\perp}\right)$ and the thesis follows immediately.

If $L$ is not surjective, $O \in E(L)=L\left(S^{n-1}\right)$ and $\mathbf{d}(L)=0=d(O, E(L))$.

Lemma 3.2. Suppose $X$ and $Y$ are compact, convex subsets of $\mathbb{R}^{p}$ with frontier $\partial X$ and $\partial Y$. Then $D(\partial X, \partial Y) \leq D(X, Y)$.

Proof. Assume for a contradiction that there exists a point $x$ in one of the boundaries, say, for instance, $x \in \partial X$, such that $d(x, \partial Y)>D(X, Y)$. Let $B$ be the open ball of radius $d(x, \partial Y)$ centered at $x$, so $B \cap \partial Y=\emptyset$. But there exists a $y \in Y$ such that $d(x, y) \leq D(X, Y)<d(x, \partial Y)$. In particular $y \notin \partial Y$ and thus $Y^{0}=Y-\partial Y \neq \emptyset$. Since $B \cap Y^{0}$ is not empty, then $B \subseteq Y^{0}$.

Since $X$ is convex, there is a hyperplane $L$ through $x$ such that $X$ lies on one side of $L$. Let $l$ be the half line starting at $x$, perpendicular to $L$ and pointing away from $X$. We have that $l \cap B \subseteq Y^{0}$ and that $l \cap \partial Y \neq \emptyset$. Let $z \in l \cap \partial Y$. Since there are no points of $X$ on the same side of $L$ as $z$, then $d(z, X)=d(x, z) \geq d(x, \partial Y)>$ $D(X, Y)$, which is a contradiction.

Proposition 3.3. Let $L$ and $L^{\prime}$ be linear maps from $\mathbb{R}^{n}$ to $\mathbb{R}^{p}$. If $\left\|L-L^{\prime}\right\| \leq \epsilon$ for some $\epsilon>0$, then $\left|\mathbf{d}(L)-\mathbf{d}\left(L^{\prime}\right)\right| \leq \epsilon$.

Proof. Without loss of generality we can assume that $n \geq p$. By hypothesis, for any unit vector $u \in \mathbb{R}^{n}$ we have that $\left\|L u-L^{\prime} u\right\| \leq \epsilon$. It easily follows that $D\left(L\left(S^{n-1}\right), L^{\prime}\left(S^{n-1}\right)\right) \leq \epsilon$, and hence $D\left(E(L), E\left(L^{\prime}\right)\right) \leq \epsilon$ by Lemma 3.2. Recall that $d(x, X) \leq d(x, Y)+D(X, Y)$ for any $x \in \mathbb{R}^{p}$ and for any $X, Y \subseteq \mathbb{R}^{p}$. Hence by Lemma 3.1 it follows that $\left|\mathbf{d}(L)-\mathbf{d}\left(L^{\prime}\right)\right|=\left|d(O, E(L))-d\left(O, E\left(L^{\prime}\right)\right)\right| \leq \epsilon$.

We will need to control how much a ball can be shrunk by a submersion $g$; this will be achieved by bounding the value of the function $\mathbf{d}$ on the differential of the map $g$.

Lemma 3.4. Let $L \in \operatorname{Hom}\left(\mathbb{R}^{n}, \mathbb{R}^{p}\right)$ be surjective and denote by $L^{*} \in \operatorname{Hom}\left(\mathbb{R}^{p}, \mathbb{R}^{n}\right)$ the adjoint map of L. Let $\Theta_{L}: \mathbb{R}^{p} \rightarrow \mathbb{R}^{n}$ be the linear map defined by $\Theta_{L}=$ $L^{*} \circ\left(L \circ L^{*}\right)^{-1}$. Then

(a) $L \circ \Theta_{L}=i d$,

(b) $\operatorname{Im} \Theta_{L}=(\operatorname{ker} L)^{\perp}$,

(c) $\left\|\Theta_{L}\right\|=\frac{1}{\mathbf{d}(L)}$.

Proof. By (a) and (b), which can be trivially checked, we have

$$
\left\|\Theta_{L}\right\|=\max _{u \neq 0} \frac{\left\|\Theta_{L}(u)\right\|}{\|u\|}=\max _{v \neq 0, v \in(\operatorname{ker} L)^{\perp}} \frac{\|v\|}{\|L(v)\|}=\frac{1}{\mathbf{d}(L)} .
$$

Let $\Omega$ be an open subset of $\mathbb{R}^{n}$ and $g: \Omega \rightarrow R^{p}$ a differentiable map. If $d_{x} g$ is the differential of $g$ at $x \in \Omega$, we will set $\Lambda g(x)=\mathbf{d}\left(d_{x} g\right)$.

Observe that the function $\Lambda g(x)$ is continuous and, if $g$ is analytic, $\Lambda g(x)$ is subanalytic. 
Lemma 3.5. Let $\Omega$ be an open subset of $\mathbb{R}^{n}$ and let $g: \Omega \rightarrow \mathbb{R}^{p}$ be a differentiable map which is a submersion on an open ball $B(x, \rho) \subseteq \Omega$. Let $r>0$ and assume that $\Lambda g(y) \geq \frac{r}{\rho}$ for all $y \in B(x, \rho)$. Then $g(B(x, \rho)) \supseteq B(g(x), r)$.

Proof. Choose any $z \in B(g(x), r)$. Let $l$ be the line through $g(x)$ and $z$, and denote by $l^{+}$the half line starting from $g(x)$ and containing $z$. Since $g$ is a submersion on $B(x, \rho)$, then $M=g^{-1}(l) \cap B(x, \rho)$ is a submanifold of $B(x, \rho)$. Let $v$ be the unit tangent field to $l$ pointing in the direction $g(x)$ to $z$. Then we have a smooth vector field on $M$ given by

$$
w(y)=\Theta_{d_{y} g}(v(g(y)))
$$

By Lemma 3.4 we have

$$
0<\|w(y)\| \leq\left\|\Theta_{d_{y} g}\right\|=\frac{1}{\Lambda g(y)} \leq \rho / r \quad \forall y \in M .
$$

Then, by classical results, there exists an integral curve $\alpha:\left(t_{1}, t_{2}\right) \rightarrow M$ of the vector field $w$ such that $t_{1}<0<t_{2}, \alpha(0)=x$ and such that $\lim _{t \rightarrow t_{2}}\|\alpha(t)-x\|=\rho$. It follows that $\beta(t)=g(\alpha(t))$ is a parametrization of a segment contained in $l$ and, again by Lemma 3.4 .

$$
\frac{d \beta}{d t}=d_{\alpha(t)} g \cdot w(\alpha(t))=v .
$$

Since $v$ is a unit vector, $\beta(t)$ is an arc-length parametrization for $t \in\left[0, t_{2}\right)$. Moreover for $t \in\left[0, t_{2}\right)$ we have that

$$
\|\alpha(t)-x\| \leq \int_{0}^{t}\left\|\frac{d \alpha}{d t}(\tau)\right\| d \tau=\int_{0}^{t}\|w(\alpha(\tau))\| d \tau \leq \frac{\rho}{r} t
$$

which implies, if we let $t$ tend to $t_{2}$, that $\rho \leq \frac{\rho}{r} t_{2}$, i.e. $t_{2} \geq r$. From these facts it follows that $\operatorname{Im}(\beta) \supseteq l^{+} \cap B(g(x), r)$ and hence there exists $u \in B(x, \rho)$ such that $g(u)=z$.

\section{Algebraic approximation of analytic Zero-Sets}

The results we present in this section give sufficient conditions that ensure the possibility of algebraically approximating an analytic set $X$ implicitly defined.

If $f$ is an analytic map defined in a neighborhood of $O$, we will denote by $T^{k} f(O)$ the polynomial map whose components are the Taylor polynomials of order $k$ at $O$ of the components of $f$.

Lemma 4.1. Let $\Omega$ be an open subset of $\mathbb{R}^{n}$ and let $f: \Omega \rightarrow \mathbb{R}^{p}$ be an analytic map, with $n>p$, such that $O \in V=V(f)$. If $\Sigma=\left\{x \in V \mid\right.$ rk $\left.d_{x} f<p\right\}$, let $U=V \backslash \Sigma$ and assume that $O \in \operatorname{Der}(U)$. Then for any $s \geq 1$ there exists a positive integer $k$ with the following property: if $g: \Omega \rightarrow \mathbb{R}^{p}$ is an analytic map such that $T^{k} g(O)=T^{k} f(O)$, then

(1) $\bar{U} \leq_{s} V(g)$,

(2) $V(g) \leq_{s} V$.

Proof. Since $s$-equivalence depends only on the germs at $O$, we are allowed to identify a subanalytic set with a realization of its germ at the origin in a suitable ball $B(O, R)$, which we will shrink whenever necessary without mention. Let $s \geq 1$.

If $O \in \operatorname{Der}(\Sigma)$, by Proposition 2.8 there exists a subanalytic set $V^{\prime} \subseteq \bar{U}$ such that $V^{\prime} \cap \Sigma=\{O\}$ and $\bar{U} \leq_{s} V^{\prime}$. Otherwise we take $V^{\prime}=\bar{U}=V$. 
The subanalytic function $\Lambda f(x)$ is positive on $V^{\prime} \backslash\{O\}$; hence, by the Eojasiewicz inequality, there exists a positive constant $\beta$ such that $\Lambda f(x)>\|x\|^{\beta}$ for all $x$ in $V^{\prime} \backslash\{O\}$.

The set $\left\{(x, y) \in U \times \mathcal{H}_{s}(U) \mid \Lambda f(y)>\|x\|^{\beta}\right\}$ contains an open subanalytic neighborhood $W$ of the diagonal $\left\{(x, x) \mid x \in V^{\prime} \backslash\{O\}\right\}$.

The function $\varphi: V^{\prime} \backslash\{O\} \rightarrow \mathbb{R}$ defined by $\varphi(x)=d((x, x), \partial W)$ is subanalytic and positive. Then again by Łojasiewicz's inequality there exists $\sigma$ (and we can assume $\sigma>s$ ) such that $\varphi(x)>\|x\|^{\sigma}$ on $V^{\prime} \backslash\{O\}$. Then for all $x \in V^{\prime} \backslash\{O\}$ and for all $y \in B\left(x,\|x\|^{\sigma}\right)$ we have

$$
d((x, y),(x, x))=d(y, x)<\|x\|^{\sigma}<\varphi(x) .
$$

Hence $(x, y) \in W$; i.e. for all $x$ in $V^{\prime} \backslash\{O\}$ and for all $y \in B\left(x,\|x\|^{\sigma}\right)$ we have $\Lambda f(y)>\|x\|^{\beta}$. In particular $\Lambda f(y)>0$ and hence $d_{y} f$ is surjective for all $y \in$ $B\left(x,\|x\|^{\sigma}\right)$.

Let $q$ be an integer such that $q>\beta+1$.

If $g: \Omega \rightarrow \mathbb{R}^{p}$ is an analytic map such that $T^{q} g(O)=T^{q} f(O)$, then $T^{q-1} d_{y} g(O)$ $=T^{q-1} d_{y} f(O)$; thus we have that $\left\|d_{y} f-d_{y} g\right\| \leq\|y\|^{q-1}$ for all $y$ near enough to $O$. Then by Proposition 3.3 we have

$$
|\Lambda f(y)-\Lambda g(y)| \leq\|y\|^{q-1} .
$$

We claim that

$$
\Lambda g(y) \geq\|x\|^{\beta+1} \quad \forall x \in V^{\prime} \backslash\{O\} \text { near enough to } O, \quad \forall y \in B\left(x,\|x\|^{\sigma}\right) .
$$

To see this, assume for contradiction that there exist a sequence $x_{i} \in V^{\prime} \backslash\{O\}$ converging to $O$ and a sequence $y_{i} \in B\left(x_{i},\left\|x_{i}\right\|^{\sigma}\right)$ such that $\Lambda g\left(y_{i}\right)<\left\|x_{i}\right\|^{\beta+1}$. Thus we have

$$
\frac{\Lambda f\left(y_{i}\right)-\Lambda g\left(y_{i}\right)}{\left\|x_{i}\right\|^{\beta}}>\frac{\left\|x_{i}\right\|^{\beta}-\left\|x_{i}\right\|^{\beta+1}}{\left\|x_{i}\right\|^{\beta}}=1-\left\|x_{i}\right\| .
$$

On the other hand,

$$
\begin{aligned}
& \frac{\Lambda f\left(y_{i}\right)-\Lambda g\left(y_{i}\right)}{\left\|x_{i}\right\|^{\beta}} \leq \frac{\left\|y_{i}\right\|^{q-1}}{\left\|x_{i}\right\|^{\beta}} \leq \frac{\left(\left\|y_{i}-x_{i}\right\|+\left\|x_{i}\right\|\right)^{q-1}}{\left\|x_{i}\right\|^{\beta}} \\
& =\left(\frac{\left\|y_{i}-x_{i}\right\|}{\left\|x_{i}\right\|^{h}}+\left\|x_{i}\right\|^{1-h}\right)^{q-1} \leq\left(\left\|x_{i}\right\|^{\sigma-h}+\left\|x_{i}\right\|^{1-h}\right)^{q-1},
\end{aligned}
$$

where $h=\frac{\beta}{q-1}$. Since $\sigma>1$ and $h<1$, we have that $\frac{\Lambda f\left(y_{i}\right)-\Lambda g\left(y_{i}\right)}{\left\|x_{i}\right\|^{\beta}}$ converges to 0 , which is a contradiction.

In particular $\forall x \in V^{\prime} \backslash\{O\}$ the map $g$ is a submersion on $B\left(x,\|x\|^{\sigma}\right)$. Hence, using Lemma 3.5, we get $g\left(B\left(x,\|x\|^{\sigma}\right)\right) \supseteq B\left(g(x),\|x\|^{\sigma^{\prime}}\right)$ with $\sigma^{\prime}=\beta+1+\sigma$.

Observe that if $x \in V^{\prime} \backslash\{O\}$, the condition $T^{q} g(O)=T^{q} f(O)$ implies that

$$
\lim _{x \rightarrow O} \frac{\|g(x)\|}{\|x\|^{q}}=\lim _{x \rightarrow O} \frac{\|g(x)-f(x)\|}{\|x\|^{q}}=0 .
$$

So, if $q \geq \sigma^{\prime}$ and $x \in V^{\prime}$, the point $O$ belongs to $B\left(g(x),\|x\|^{\sigma^{\prime}}\right)$ and hence there exists $y \in B\left(x,\|x\|^{\sigma}\right)$ such that $g(y)=O$, and so $V^{\prime} \backslash\{O\} \subseteq \mathcal{H}(V(g), \sigma)$. Then by Proposition 2.6 we have $V^{\prime} \leq_{s} V(g)$ and hence $\bar{U} \leq_{s} V(g)$.

Now by the Eojasiewicz inequality there exists a real positive constant $\alpha$ such that $\|f(x)\| \geq d(x, V)^{\alpha}$ for all $x$ near enough to $O$; then for $x \notin \mathcal{H}(V, \sigma)$ we have that $\|f(x)\| \geq\|x\|^{\alpha \sigma}$. 
Let $k$ be an integer such that $k \geq \max \left\{\alpha \sigma, \sigma^{\prime}\right\}$.

If $g: \Omega \rightarrow \mathbb{R}^{p}$ is an analytic map such that $T^{k} g(O)=T^{k} f(O)$, then

$$
\lim _{x \rightarrow O} \frac{\|f(x)-g(x)\|}{\|x\|^{\alpha \sigma}}=0 .
$$

We have that $V(g) \backslash\{O\} \subseteq \mathcal{H}(V, \sigma)$; otherwise there would exist a sequence of points $y_{i} \neq O$ converging to $O$ such that $y_{i} \in V(g) \backslash \mathcal{H}(V, \sigma)$ and hence

$$
\lim _{i \rightarrow \infty} \frac{\left\|f\left(y_{i}\right)-g\left(y_{i}\right)\right\|}{\left\|y_{i}\right\|^{\alpha \sigma}}=\lim _{i \rightarrow \infty} \frac{\left\|f\left(y_{i}\right)\right\|}{\left\|y_{i}\right\|^{\alpha \sigma}} \geq 1,
$$

which is a contradiction. Then by Proposition 2.6 we get that $V(g) \leq_{s} V$.

As a direct application of Lemma 4.1 we get

Corollary 4.2. Let $\Omega$ be an open subset of $\mathbb{R}^{n}$ and let $f: \Omega \rightarrow \mathbb{R}^{p}$ be an analytic map such that $O$ is a nonisolated point in $V=V(f)$. If $\Sigma=\left\{x \in V \mid \operatorname{rk} d_{x} f<p\right\}$, assume that $\overline{V \backslash \Sigma}=V$. Then, for any $s \geq 1$ there exists a positive integer $k$ such that if $g=T^{k} f(O)$, then $V \sim_{s} V(g)$.

The previous result giving sufficient conditions to approximate an analytic set by means of an algebraic one can be extended to the following more general situation.

Theorem 4.3. Let $\Omega$ be an open subset of $\mathbb{R}^{n}$ and let $X \subset \Omega$ be an analytic set such that $O$ is a nonisolated point in $X$; assume that there exist finitely many analytic maps $f_{i}: \Omega \rightarrow \mathbb{R}^{p_{i}}, i=1, \ldots, m$, with $n>p_{i}$ for all $i$, such that $X=\bigcup_{i=1}^{m} V\left(f_{i}\right)$. If $\Sigma_{i}=\left\{x \in V\left(f_{i}\right) \mid \operatorname{rk} d_{x} f_{i}<p_{i}\right\}$ and $U_{i}=V\left(f_{i}\right) \backslash \Sigma_{i}$, assume that $X=\bigcup_{i=1}^{m} \overline{U_{i}}$. Then, for any $s \geq 1$ there exists an algebraic set $Y \subseteq \mathbb{R}^{n}$ such that $X \sim_{s} Y$.

Proof. Since the problem is local, we can assume that $O \in \operatorname{Der}\left(U_{i}\right)$ for all $i$. For each $i \in\{1, \ldots, m\}$ let $k_{i}$ be a positive integer found as in Lemma 4.1 and let $g_{i}=T^{k_{i}} f_{i}(O)$. Then $V\left(g_{i}\right) \leq_{s} V\left(f_{i}\right)$ and $\overline{U_{i}} \leq_{s} V\left(g_{i}\right)$. Hence by Proposition 2.7 we have $\bigcup_{i=1}^{m} V\left(g_{i}\right) \leq_{s} \bigcup_{i=1}^{m} V\left(f_{i}\right)=X$ and $X=\bigcup_{i=1}^{m} \overline{U_{i}} \leq_{s} \bigcup_{i=1}^{m} V\left(g_{i}\right)$. Thus it suffices to take $Y=\bigcup_{i=1}^{m} V\left(g_{i}\right)$.

The following example shows that Theorem 4.3 applies to a class of analytic sets which strictly contains the one considered in Corollary 4.2

Example 4.4. Let $g(z)=1-\cos z$ and consider the analytic function $f: \mathbb{R}^{3} \rightarrow \mathbb{R}$ defined by $f(x, y, z)=z y^{2}-(x+g(z))^{2}$. Corollary 4.2 cannot be applied to the analytic (and not algebraic) set $X=V(f)$ because $\Sigma=\left\{(x, y, z) \in \mathbb{R}^{3} \mid y=\right.$ $0, x+g(z)=0\}$ and $\overline{X \backslash \Sigma} \neq X$. On the other hand, if we set $f_{1}=f, f_{2}(x, y, z)=$ $(y, x+g(z))$ and we use the notation of Theorem 4.3 , we have that $X=\overline{U_{1}} \cup \overline{U_{2}}$ and thus $X$ can be algebraically approximated to any order.

\section{Algebraic approximation of parametrized sets}

In this section we give some results concerning algebraic approximation of images of real analytic maps.

Lemma 5.1. Let $\Omega$ be an open subset of $\mathbb{R}^{d}$ containing $O$ and let $F: \Omega \rightarrow \mathbb{R}^{n}$ be an analytic map. Assume that there exists a relatively compact neighborhood $W$ of $O$ such that $\bar{W} \subset \Omega$ and such that $F^{-1}(O) \cap \bar{W}=\{O\}$. Then for any $s \geq 1$ there exists a positive integer $k$ with the following property: if $G: \Omega \rightarrow \mathbb{R}^{n}$ is an 
analytic map such that $T^{k} G(O)=T^{k} F(O)$, then there exists a neighborhood $W^{\prime}$ of $O$ contained in $W$ such that $F(W)$ is s-equivalent to $G\left(W^{\prime}\right)$ at $O$.

Proof. At first let us observe that for all open neighborhoods $W^{\prime}$ of $O$ contained in $W$ the germs $F(W)_{O}$ and $F\left(W^{\prime}\right)_{O}$ coincide. Otherwise there would exist a sequence of points $y_{i}=F\left(x_{i}\right)$ converging to $O$ with $x_{i} \in W \backslash W^{\prime}$; by compactness we can assume that $\left\{x_{i}\right\}$ converges to some point $x_{0} \in \bar{W} \backslash W^{\prime}$. So $x_{0} \neq O$ and $F\left(x_{0}\right)=O$, in contradiction with our hypothesis.

Thus it will suffice to find for any $s$ an open neighborhood $W^{\prime}$ of $O$ contained in $W$ such that $F\left(W^{\prime}\right)$ is s-equivalent to $G\left(W^{\prime}\right)$ at $O$.

By Lojasiewicz's inequality there exists a real $\alpha>0$ such that $\|F(x)\| \geq\|x\|^{\alpha}$ for all $x \in W$. Let $\sigma>s$ and let $k$ be an integer such that $k \geq \alpha \sigma$. If $G: \Omega \rightarrow \mathbb{R}^{n}$ is an analytic map such that $T^{k} G(O)=T^{k} F(O)$, then

$$
\lim _{x \rightarrow O} \frac{\|F(x)-G(x)\|}{\|F(x)\|} \leq \lim _{x \rightarrow O} \frac{\|F(x)-G(x)\|}{\|F(x)\|^{\sigma}} \leq \lim _{x \rightarrow O} \frac{\|F(x)-G(x)\|}{\|x\|^{k}}=0 .
$$

It follows that $\lim _{x \rightarrow O} \frac{\|G(x)\|}{\|F(x)\|}=1$. Then there exists an open neighborhood $W^{\prime}$ of $O$ contained in $W \cap \Omega$ such that $\|G(x)\| \geq \frac{1}{2}\|x\|^{\alpha}$ for all $x \in \overline{W^{\prime}}$; in particular $G^{-1}(O) \cap \overline{W^{\prime}}=\{O\}$.

By (5.1) there exists an open neighborhood $U$ of $O$ contained in $W^{\prime}$ such that

$$
d\left(F(x), G\left(W^{\prime}\right)\right) \leq\|F(x)-G(x)\|<\|F(x)\|^{\sigma} \quad \forall x \in U \backslash\{O\} ;
$$

hence $F(x) \in \mathcal{H}\left(G\left(W^{\prime}\right), \sigma\right)$ for all $x \in U \backslash\{O\}$, i.e. $F(U \backslash\{O\}) \subseteq \mathcal{H}\left(G\left(W^{\prime}\right), \sigma\right)$.

By the initial argument $F(U)_{O}=F\left(W^{\prime}\right)_{O}$; hence there exists $R>0$ such that $F\left(W^{\prime}\right) \cap B(O, R) \backslash\{O\} \subseteq \mathcal{H}\left(G\left(W^{\prime}\right), \sigma\right)$ and thus, by Proposition 2.6, we have that $F\left(W^{\prime}\right) \leq_{s} G\left(W^{\prime}\right)$.

Since $\lim _{x \rightarrow O} \frac{\|G(x)\|}{\|F(x)\|}=1$, by (5.1) we have that $\lim _{x \rightarrow O} \frac{\|F(x)-G(x)\|}{\|G(x)\|^{\sigma}}=0$. Thus, if we use the same argument as above exchanging the role of $F\left(W^{\prime}\right)$ and $G\left(W^{\prime}\right)$, we achieve the thesis.

Proposition 5.2. Let $X$ be a subanalytic subset of $\mathbb{R}^{n}$, with $O \in \operatorname{Der}(X)$. Assume there exist a real analytic manifold $M$, a relatively compact open subset $W$ of $M, a$ neighborhood $U$ of $O$ in $\mathbb{R}^{n}$ and an analytic map $F: M \rightarrow \mathbb{R}^{n}$ such that $F^{-1}(O) \cap \bar{W}$ consists of a single point $P$ and $X \cap U=F(W)$. Then for any $s \geq 1$ there exists an algebraic subset $Y$ of $\mathbb{R}^{n}$ such that $X \sim_{s} Y$.

Proof. We can assume that $M=\mathbb{R}^{d}$ and $P=O$. Let $k$ be a positive integer found as in Lemma 5.1 and let $G=T^{k} F(O)$. Then by Lemma 5.1 there exists a neighborhood $W^{\prime}$ of $O$ contained in $W$ such that $X$ is $s$-equivalent at $O$ to the semialgebraic set $G\left(W^{\prime}\right)$. By Theorem 2.2 there exists an algebraic subset $Y$ of $\mathbb{R}^{n}$ which is $s$-equivalent to $G\left(W^{\prime}\right)$ and hence to $X$.

A first consequence of the previous proposition concerns one-dimensional subanalytic sets:

Proposition 5.3. Let $X$ be a closed subanalytic subset of $\mathbb{R}^{n}$ of dimension 1 , with $O \in \operatorname{Der}(X)$. Then for any $s \geq 1$ there exists an algebraic subset $Y$ of $\mathbb{R}^{n}$ such that $X \sim_{s} Y$ at $O$. 
Proof. Since the problem is local, we can assume that $X$ is compact. By the Uniformization Theorem for subanalytic sets (see for instance [BM]) there exist a compact real analytic manifold $M$ of dimension 1 and a proper real analytic map $\phi: M \rightarrow \mathbb{R}^{n}$ such that $\phi(M)=X$. Then we can assume that $\phi^{-1}(O)$ consists of finitely many points $P_{1}, \ldots, P_{m}$. Let us consider an open cover $W_{1}, \ldots, W_{m}$ of $M$ such that $\overline{W_{i}} \subset M \backslash \bigcup_{j \neq i} P_{j}$ for all $i$. By Proposition 5.2 for each $i$ there exists an algebraic $Y_{i}$ such that $\phi\left(W_{i}\right) \sim_{s} Y_{i}$. Since $X=\bigcup_{i=1}^{m} \phi\left(W_{i}\right)$, the thesis follows from Proposition 2.7

From the previous result we can easily deduce the algebraic approximability for implicitly defined analytic surfaces in $\mathbb{R}^{3}$ :

Corollary 5.4. Let $\Omega$ be an open subset of $\mathbb{R}^{3}$ containing $O, f: \Omega \rightarrow \mathbb{R}$ be an analytic map and let $X=V(f)$. Assume that $\operatorname{dim}_{O} X=2$ and that $d_{x} f$ is not identically zero on each irreducible component of $X$ through $O$. Then for any $s \geq 1$ there exists an algebraic subset $Y$ of $\mathbb{R}^{3}$ such that $X \sim_{s} Y$.

Proof. Let $\Sigma$ be the set of points of $X$ where $d_{x} f$ vanishes and let $U=X \backslash \Sigma$. Since by hypothesis $\Sigma$ has dimension at most 1 , by Proposition 5.3 there exists an algebraic set $Z_{1}$ such that $\Sigma$ is $s$-equivalent to $Z_{1}$. By Lemma 4.1 there exists an algebraic set $Z_{2}$ such that $Z_{2} \leq_{s} X$ and $\bar{U} \leq_{s} Z_{2}$. Then $Y:=Z_{1} \cup Z_{2}$ is s-equivalent to $X$.

The algebraic approximation of real analytic curves could also be obtained as a direct consequence of Proposition 5.2 using the real version of the well-known Puiseux theorem (see [M]) which assures that each branch at a point, say $O$, of a real analytic curve contained in $\mathbb{R}^{n}$ admits a local parametric representation of the form $F(t)=\left( \pm t^{k}, f(t)\right)$ where $f$ is a real analytic map with $f(0)=O$.

The Abhyankar-Jung theorem (see [Ab]) generalizes Puiseux's theorem to quasiordinary hypersurface singularities. Recall (see for instance $\mathrm{Ar}$ ) that a $d$-dimensional complex analytic singularity $\left(X, x_{0}\right)$ is called quasi-ordinary if there exists a finite map of analytic germs $\pi:\left(X, x_{0}\right) \rightarrow\left(\mathbb{C}^{d}, O\right)$ whose discriminant locus has a normal crossing at $O$. The Abhyankar-Jung theorem has been further generalized by Aroca $(\underline{\mathrm{Ar}})$ to the case of complex analytic subsets of $\mathbb{C}^{n}$ of higher codimension. We will use Aroca's result in the proof of the following theorem.

Theorem 5.5. Let $X \subset \mathbb{R}^{n}$ be an analytic set such that $O \in X$ and such that the germ $X_{O}$ has pure dimension d. Assume that its complexification $X_{\mathbb{C}}$ has $O$ as a quasi-ordinary singular point. Then for any $s \geq 1$ there exists a real algebraic set $Y$ such that $X \sim_{s} Y$ at $O$.

Proof. By $\mathrm{Ar}$ there exist a neighborhood $U$ of $O$ in $\mathbb{C}^{n}$, a neighborhood $\Omega$ of $O$ in $\mathbb{C}^{d}$, an integer $k$ and an analytic map $g: \Omega \rightarrow \mathbb{C}^{n-d}$ such that

$$
X_{\mathbb{C}} \cap U=\left\{\left(u_{1}^{k}, \ldots, u_{d}^{k}, g\left(u_{1}, \ldots, u_{d}\right)\right) \mid\left(u_{1}, \ldots, u_{d}\right) \in \Omega\right\} .
$$

Up to shrinking $U$, we can assume that $X_{\mathbb{C}} \cap U$ has pure dimension $d$.

We claim that for $i=1, \ldots, h$, there exist $\Omega_{i}$ open in $\mathbb{R}^{d}$ and $f_{i}: \Omega_{i} \rightarrow \mathbb{R}^{n-d}$ a real analytic map such that

$$
X \cap U=\bigcup_{i=1}^{h}\left\{\left(\epsilon_{i, 1} t_{1}^{k}, \ldots, \epsilon_{i, d} t_{d}^{k}, f_{i}\left(t_{1}, \ldots, t_{d}\right)\right) \mid\left(t_{1}, \ldots, t_{d}\right) \in \Omega_{i}\right\},
$$

where $\epsilon_{i, j} \in\{1,-1\}$ for $j=1, \ldots, d$. 
To prove this, observe that the point $\left(u_{1}^{k}, \ldots, u_{d}^{k}, g\left(u_{1}, \ldots, u_{d}\right)\right)$ lies in $X \cap U$ if and only if there exist $2 k$-th roots of unity $\xi_{1}, \ldots, \xi_{d}$ such that $u_{i}=\xi_{i} t_{i}$ with $t_{i} \in \mathbb{R}$ and $g\left(u_{1}, \ldots, u_{d}\right) \in \mathbb{R}^{n-d}$.

Let $\Xi=\left\{\xi=\left(\xi_{1}, \ldots, \xi_{d}\right) \in \mathbb{C}^{d} \mid \xi_{j}^{2 k}=1 \quad \forall j\right\}$. For each $\xi \in \Xi$ consider the set

$$
V_{\xi}=\left\{\left(t_{1}, \ldots, t_{d}\right) \in \mathbb{R}^{d} \mid\left(\xi_{1} t_{1}, \ldots, \xi_{d} t_{d}\right) \in \Omega, g\left(\xi_{1} t_{1}, \ldots, \xi_{d} t_{d}\right) \in \mathbb{R}^{n-d}\right\}
$$

Let $g^{\xi}\left(t_{1}, \ldots, t_{d}\right)=g\left(\xi_{1} t_{1}, \ldots, \xi_{d} t_{d}\right)$; then $g^{\xi}=g^{\xi}{ }_{1}+i g^{\xi}{ }_{2}$ with $g^{\xi}{ }_{1}, g^{\xi}$ real analytic maps. Thus

$$
V_{\xi}=\left\{\left(t_{1}, \ldots, t_{d}\right) \in \mathbb{R}^{d} \mid\left(\xi_{1} t_{1}, \ldots, \xi_{d} t_{d}\right) \in \Omega, g^{\xi}{ }_{2}\left(t_{1}, \ldots, t_{d}\right)=O\right\}
$$

hence $V_{\xi}$ is analytic in an open subset of $\mathbb{R}^{d}$.

Denote by $\psi_{\xi}: V_{\xi} \rightarrow \mathbb{R}^{n}$ the restriction to $V_{\xi}$ of the analytic map defined by

$$
\left(t_{1}, \ldots, t_{d}\right) \rightarrow\left(\xi_{1}{ }^{k} t_{1}{ }^{k}, \ldots, \xi_{d}{ }^{k} t_{d}^{k}, g^{\xi}{ }_{1}\left(t_{1}, \ldots, t_{d}\right)\right)
$$

Then $X \cap U=\bigcup_{\xi \in \Xi} \psi_{\xi}\left(V_{\xi}\right)$. Let

$$
\Xi^{\prime}=\left\{\xi \in \Xi \mid \operatorname{dim} \psi_{\xi}\left(V_{\xi}\right)=d\right\}=\left\{\xi^{(1)}, \ldots, \xi^{(h)}\right\}
$$

Observe that $V_{\xi}$ is an open subset of $\mathbb{R}^{d}$ for each $\xi \in \Xi^{\prime}$ and that $X \cap U=$ $\bigcup_{\xi \in \Xi^{\prime}} \psi_{\xi}\left(V_{\xi}\right)$ since $X \cap U$ is equidimensional.

Setting $\epsilon_{i, j}=\left(\xi_{j}^{(i)}\right)^{k}, \Omega_{i}=V_{\xi^{(i)}}$ and $f_{i}\left(t_{1}, \ldots, t_{d}\right)=g\left(\xi_{1}^{(i)} t_{1}, \ldots, \xi_{d}^{(i)} t_{d}\right)$, we get a proof of our claim. Hence we achieve the thesis using Proposition 5.2 and Proposition 2.7.

\section{REFERENCES}

[Ab] S. Abhyankar: On the ramification of algebraic functions. Amer. J. Math., vol. 77 (1955), pp. 575-592. MR0071851 (17:193c)

[Ar] F. Aroca: Puiseux parametric equations of analytic sets. Proc. Amer. Math. Soc., vol. 132, n. 10 (2004), pp. 3035-3045 (electronic). MR2063125 (2005c:32032)

[BM] E. Bierstone, P. Milman: Semianalytic and subanalytic sets. Inst. Hautes Études Sci. Publ. Math., vol. 67 (1988), pp. 5-42. MR.972342 (89k:32011)

[B1] M. Bilski: Approximation of analytic sets by Nash tangents of higher order. Math. Z., vol. 256, n. 4 (2007), pp. 705-716. MR.2308884 (2008h:32009)

[B2] M. BILSKI: Approximation of analytic sets with proper projection by algebraic sets. arXiv:0905.1881v1[math.CV] (2009).

[FFW] M. Ferrarotti, E. Fortuna And L. Wilson: Local approximation of semialgebraic sets. Ann. Scuola Norm. Sup. Pisa Cl. Sci. (5), vol. I, n. 1 (2002), pp. 1-11. MR.1994799 (2004f:14083)

[H] H. Hironaka: Stratification and flatness. In: Real and complex singularities (Proc. Ninth Nordic Summer School/NAVF Sympos. Math., Oslo, 1976), Sijthoff and Noordhoff, Alphen aan den Rijn, 1977, pp. 199-265. MR0499286 (58:17187)

[KR] K. Kurdyka, G. Raby: Densité des ensembles sous-analytiques. Ann. Inst. Fourier (Grenoble), vol. 39 (1989), pp. 753-771. MR1030848 (90k:32026) 
[M] J. Milnor: Singular points of complex hypersurfaces. Annals of Mathematics Studies, No. 61, Princeton University Press, Princeton, N.J.; University of Tokyo Press, Tokyo, 1968. MR0239612(39:969)

[TW] D. Trotman, L. Wilson: Stratifications and finite determinacy. Proc. London Math. Soc. (3), vol. 78, n. 2 (1999), pp. 334-368. MR.1665246 (2000h:58069)

Dipartimento di Matematica, Politecnico di Torino, Corso Duca degli Abruzzi 24, I-10129 TORINo, ITALY

E-mail address: ferrarotti@polito.it

Dipartimento di Matematica, Università di Pisa, Largo B. Pontecorvo 5, I-56127 Pisa, ITALY

E-mail address: fortuna@dm.unipi.it

Department of Mathematics, University of Hawait, Manoa, Honolulu, Hawail 96822

E-mail address: les@math.hawaii.edu 